\title{
Health-Related Quality of Life in the Gender, Race, And Clinical Experience Trial
}

\author{
Judith Feinberg, ${ }^{1}$ Michael Saag, ${ }^{2}$ Kathleen Squires, ${ }^{3}$ Judith Currier, ${ }^{4}$ Robert Ryan, ${ }^{5}$ \\ Bruce Coate, ${ }^{5}$ and Joseph Mrus ${ }^{6}$
}

\author{
${ }^{1}$ Department of Internal Medicine, University of Cincinnati College of Medicine, 231 Albert Sabin Way, Cincinnati, OH 45267, USA \\ ${ }^{2}$ Division of Infectious Disease, Department of Medicine, University of Alabama at Birmingham, Birmingham, AL 35294, USA \\ ${ }^{3}$ Division of Infectious Diseases and Environmental Medicine, Jefferson Medical College of Thomas Jefferson University, \\ Philadelphia, PA 19107, USA \\ ${ }^{4}$ David Geffen School of Medicine, University of California, Los Angeles, Los Angeles, CA 90035, USA \\ ${ }^{5}$ Medical Affairs Biometrics, Tibotec Inc., Titusville, NJ 08560, USA \\ ${ }^{6}$ Clinical Affairs, Tibotec Therapeutics, Titusville, NJ 08560, USA
}

Correspondence should be addressed to Judith Feinberg, feinbej@ucmail.uc.edu

Received 30 March 2011; Accepted 1 June 2011

Academic Editor: Magnus Gisslén

Copyright ( $\odot 2011$ Judith Feinberg et al. This is an open access article distributed under the Creative Commons Attribution License, which permits unrestricted use, distribution, and reproduction in any medium, provided the original work is properly cited.

\begin{abstract}
Background. We report health-related QoL (HRQoL) from GRACE ( $\underline{\text { Gender, }}$ Race, $\underline{\text { And }}$ Clinical Experience) study by sex and race over 48 weeks. Methods. 429 treatment-experienced adults (HIV-1 RNA $\geq 1000$ copies $/ \mathrm{mL}$ ) received darunavir/ritonavir $600 / 100 \mathrm{mg}$ twice daily plus an appropriate background regimen. QoL was measured by the Functional Assessment of HIV Infection (FAHI) questionnaire. Results. $67 \%$ women and $77 \%$ men, including $67.4 \%$ black, $76.0 \%$ Hispanic, and $73.8 \%$ white patients, completed the trial. Baseline total FAHI scores were similar between sexes and races. Total FAHI of the entire population improved by Week $4(P<.05)$; near-maximum changes obtained by Week 12 were maintained through Week 48 . Women and black patients demonstrated larger improvements in total FAHI versus men, and Hispanic and white patients, respectively. Conclusion. HRQoL improved in all sex and racial/ethnic groups. Sex-based and race-based differences in improvements in FAHI subscales may provide insight into subtle differences of HIV-1 and treatment on HRQoL in different populations.
\end{abstract}

\section{Introduction}

Improvements in antiretroviral (ARV) therapy have increased the life expectancy of patients with HIV-1 infection, and HIV is now considered a manageable chronic disease [1]. The maintenance of a high quality of life (QoL), as well as optimization of physical, emotional, and social health, is an important aspect of disease management for HIV-1-infected patients. Health-related quality of life (HRQoL) measures are used to quantify the physical and mental aspects of HIV1 infection that can impact a patient's overall well-being. Additionally, several studies have demonstrated a correlation between HRQoL and survival of HIV-1-infected patients [2-4], highlighting the need for monitoring and optimizing HRQoL. The GRACE (Gender, Race, And Clinical Experience) study was designed to investigate sex-based and race-based differences in outcomes with darunavir/ritonavir$(\mathrm{DRV} / \mathrm{r}-)$ based therapy in treatment-experienced patients. The primary outcomes of the GRACE study have been reported [5]. Here, we report the HRQoL results from GRACE by sex and race over 48 weeks.

\section{Methods}

2.1. Study Design and Patients. GRACE was a 48-week, openlabel, Phase IIIb study conducted at 65 study sites across the United States, Canada, and Puerto Rico. Treatmentexperienced adults (HIV-1 RNA $\geq 1000$ copies $/ \mathrm{mL}$ ) received DRV/r 600/100 mg twice daily plus an appropriate ARV background regimen, chosen on the basis of resistance testing [5]. Details of the study design and the primary outcomes of the GRACE study have been reported elsewhere [5]. Human 
experimentation guidelines of the US Department of Health and Human Services were followed in the conduct of this clinical research, the research protocol was reviewed and approved by institutional review boards for all 65 study sites, and all participants provided written informed consent.

2.2. Study Evaluations. Health-related QoL was measured by the Functional Assessment of HIV Infection (FAHI) questionnaire, which contained 47 questions measuring five functional subscales [6, 7]: physical (PWB), emotional (EWB), functional and global (FGWB), and social (SWB) well-being, as well as cognitive functioning (CF). The range of possible scores for each subscale was as follows: $\mathrm{PWB}$ and EWB, 0 to 40; FGWB, 0 to 52; SWB, 0 to 32; CF, 0 to 12 . The total FAHI score, with possible scores ranging from 0 to 176 , was calculated as the sum of all five subscale values, with higher scores indicating better results. Each subject completed the FAHI questionnaire at the beginning of the study visit, before other assessments, at study entry, and at weeks $4,12,24$, and 48 or at early withdrawal. Questionnaires completed at early withdrawal visits were not included in the analyses. The time recall period was the previous 7 days. Post hoc analyses were conducted in order to investigate factors that were associated with improvement in total FAHI score over 48 weeks.

2.3. Statistical Analyses. Analyses were performed on the observed population. Imputed values were derived for each of the five subscales of the FAHI questionnaire. Imputed values were calculated if at least $50 \%$ of the items in that subscale were present using the mean of the other items in the subscale, rounded to the nearest integer; if greater than $50 \%$ of the items in a given subscale were missing, no imputation was calculated. Results from intention-totreat-last-observation-carried-forward analyses were similar to results obtained from the observed population and are not presented here.

In the post hoc analysis that investigated factors associated with improvement in total FAHI score, 18 covariates were evaluated using a repeated measure analysis; covariates significant at the $P<.10$ level were considered for the multivariate analysis. The multivariate analysis was performed using a forward stepwise selection with stay criteria of $P<$ .05. If two or more covariates selected from the univariate analyses were highly correlated, only the most significant was considered for inclusion in the multivariate models to avoid issues with collinearity.

\section{Results}

3.1. Patient Population and Baseline Characteristics. The GRACE study enrolled 429 patients, of whom 66.9\% were women, $61.5 \%$ were black, $22.4 \%$ were Hispanic, and $15.2 \%$ were white. In the intent-to-treat population, overall, 53.4\% achieved virologic response (HIV RNA $<50$ copies $/ \mathrm{mL}$; women, 50.9\%; men, 58.5\%; black, 48.5\%; Hispanic, $61.5 \%$; white, $60.0 \%)[5,8]$. In the time-to-loss of virologic response-non-virologic-failure censored analysis of the overall population that censored patients who discontinued for reasons other than virologic failure, $73.2 \%$ of patients (women, 73.0\%; men, 73.5\%; black, 68.8\%; Hispanic, 79.7\%; white, $78.0 \%$ ) achieved virologic response $[5,8]$.

The HRQoL analysis included the 193 women (67\%) and 109 men $(77 \%)$ who completed the trial (Figure 1(a)). By race, 178 black (67.4\%), 73 Hispanic (76.0\%), and 48 white $(73.8 \%)$ patients completed the trial (Figure 1(b)). Women had a higher rate of discontinuation compared with men, mostly due to reasons other than virologic failure, and black patients had a higher rate of discontinuation compared with Hispanic and white patients, mostly due to loss to followup and other reasons (Figures 1(a) and 1(b)).

At baseline, women were younger and had a higher average body mass index than men (Table 1). Men and black patients had more advanced disease at baseline than women and Hispanic or white patients, respectively, with lower median CD4+ counts and higher proportions of US Centers for Disease Control and Prevention Class C disease (Table 1). Four patients who self-identified as Asian or other race were not included in the analyses by race due to their low numbers.

\subsection{Health-Related Quality of Life: Total Functional Assess-} ment of HIV Infection Score. At baseline, the total FAHI scores for the overall population ranged from 26 to 176 . Women had slightly lower total FAHI scores compared with men (116.8 versus 120.8), and Hispanic patients had slightly lower total scores compared with black or white patients (114.1, 119.5, and 119.5, resp.; Figure 2); neither sex-based nor race-based differences in baseline scores were statistically significant. The total FAHI score of the entire GRACE population improved significantly by Week 4 (Figure 2(a)). Near-maximum changes were achieved by Week 12 and were maintained through Week 48. Similar patterns of improvement were observed for both sexes and all races (Figures 2(b) and 2(c)). Women demonstrated larger improvements in total FAHI scores compared with men, and black patients demonstrated larger improvements compared with Hispanic and white patients. Overall, only 3\% of the subscales were imputed for missing data.

The post hoc repeated measure analysis to investigate factors associated with improved FAHI over 48 weeks identified five factors with $P<.10$ (Table 2) that were considered for the multivariate analysis. The post hoc multivariate analysis subsequently identified lower baseline FAHI score, lower baseline CD4+ cell count, confirmed virologic response, and analysis time point as significantly associated with improvement in FAHI score over 48 weeks (Table 3). Notably, neither sex nor race was found to be significant in the final model, and there was no interaction between the two variables.

In a post hoc sensitivity analysis, it was noted that patients with lower baseline FAHI scores were significantly more likely to discontinue $(P=.044)$ than patients with higher baseline HRQoL scores. To assess the impact of discontinuations, an additional post hoc sensitivity analysis evaluated only patients who completed the trial. When patients who discontinued the trial were excluded and only patients who completed the trial were analyzed, the 


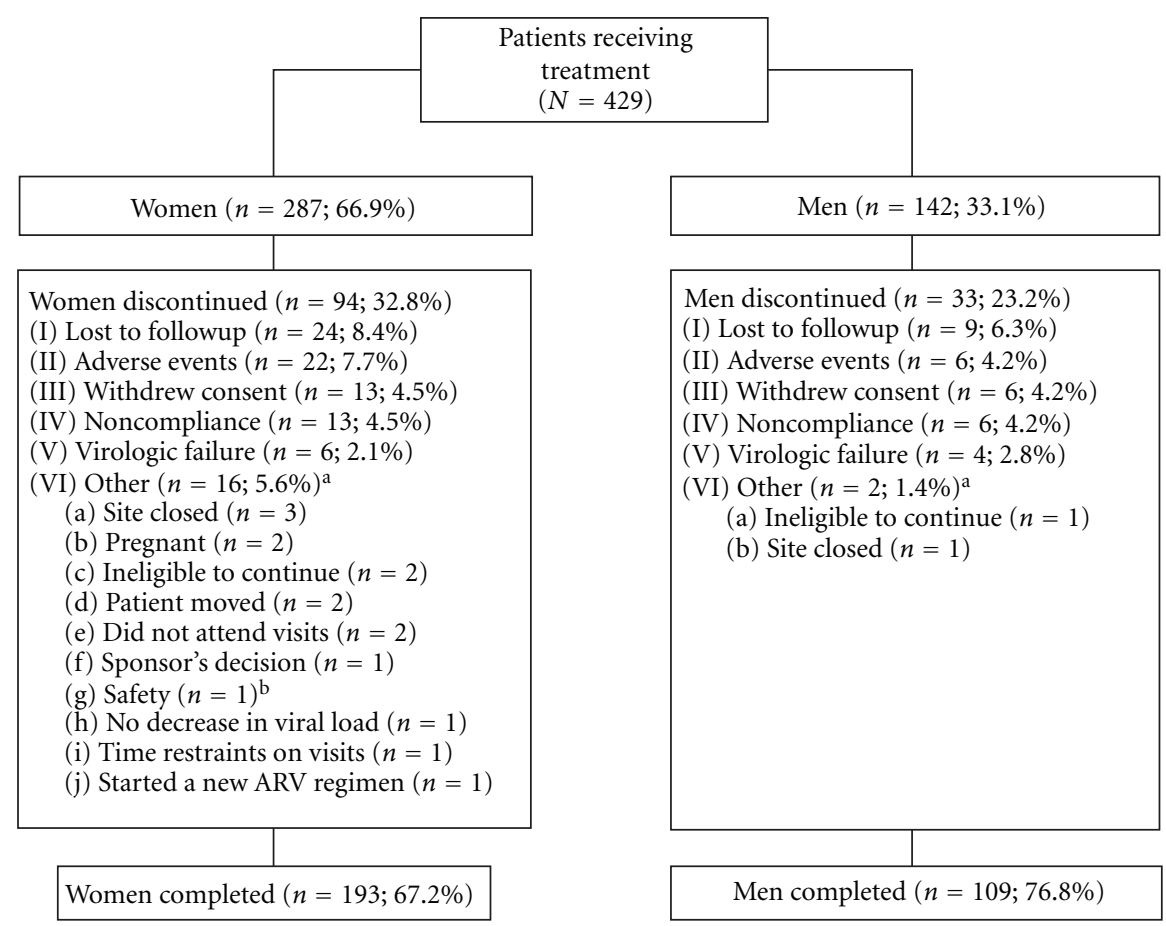

(a)

\begin{tabular}{|c|c|c|}
\hline & $\begin{array}{l}\text { Patients receiving } \\
\text { treatment } \\
(N=429)\end{array}$ & \\
\hline & \multirow{2}{*}{$\begin{array}{c}\text { Patients included in } \\
\text { analysis } \\
(n=425) \\
\end{array}$} & \multirow{2}{*}{$\begin{array}{l}\text { Patients excluded from analysis } \\
\text { (self-identified as Asian or Other) } \\
\qquad(n=4)\end{array}$} \\
\hline & & \\
\hline $\begin{array}{c}\text { Black patients } \\
(n=264 ; 61.5 \%)\end{array}$ & $\begin{array}{l}\text { White patients } \\
(n=65 ; 15.2 \%)\end{array}$ & $\begin{array}{l}\text { Hispanic patients } \\
(n=96 ; 22.4 \%)\end{array}$ \\
\hline 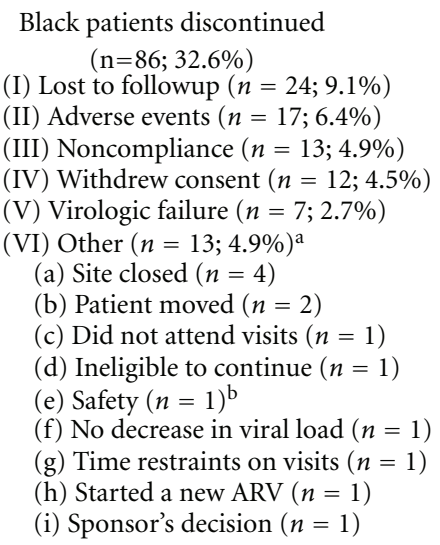 & $\begin{array}{l}\text { White patients discontinued } \\
\quad(n=17 ; 26.2 \%) \\
\text { (I) Lost to followup }(n=3 ; 4.6 \%) \\
\text { (II) Adverse events }(n=5 ; 7.7 \%) \\
\text { (III) Noncompliance }(n=2 ; 3.1 \%) \\
\text { (IV) Withdrew consent }(n=3 ; 4.6 \%) \\
\text { (V) Virologic failure }(n=2 ; 3.1 \%) \\
\text { (VI) Other }(n=2 ; 3.1 \%)^{\mathrm{a}} \\
\text { (a) Pregnant }(n=1) \\
\text { (b) Ineligible to continue }(n=1)\end{array}$ & $\begin{array}{l}\text { Hispanic patients discontinued } \\
\quad(n=23 ; 24.0 \%) \\
\text { (I) Lost to followup }(n=5 ; 5.2 \%) \\
\text { (II) Adverse events }(n=6 ; 6.3 \%) \\
\text { (III) Noncompliance }(n=4 ; 4.2 \%) \\
\text { (IV) Withdrew consent }(n=4 ; 4.2 \%) \\
\text { (V) Virologic failure }(n=1 ; 1.0 \%) \\
\text { (VI) Other }(n=3 ; 3.1 \%)^{\mathrm{a}} \\
\text { (a) Pregnant }(n=1) \\
\text { (b) Ineligible to continue }(n=1) \\
\text { (c) Did not attend visits }(n=1)\end{array}$ \\
\hline $\begin{array}{l}\text { Black patients completed } \\
\quad(n=178 ; 67.4 \%)\end{array}$ & $\begin{array}{l}\text { White patients completed } \\
\qquad(n=48 ; 73.8 \%)\end{array}$ & $\begin{array}{l}\text { Hispanic patients completed } \\
\quad(n=73 ; 76.0 \%)\end{array}$ \\
\hline
\end{tabular}

(b)

Figure 1: Study disposition by (a) sex and (b) race. " "Other" classification was selected by the investigator as reason for discontinuation. ${ }^{b}$ Older patient taking too many concomitant medications; ARV: antiretroviral. 
TABLE 1: Baseline demographics and disease characteristics by sex and race ${ }^{\mathrm{a}}$.

\begin{tabular}{|c|c|c|c|c|c|}
\hline Parameter & $\begin{array}{l}\text { Women } \\
n=287\end{array}$ & Men $n=142$ & $\begin{array}{c}\text { Black } \\
n=264\end{array}$ & $\begin{array}{c}\text { Hispanic } \\
n=96\end{array}$ & $\begin{array}{l}\text { White } \\
n=65\end{array}$ \\
\hline Age, mean (SE), years & $41.7(0.63)$ & $45.2(0.75)$ & $43.0(0.62)$ & $40.3(1.05)$ & $45.5(1.13)$ \\
\hline \multicolumn{6}{|l|}{ Gender, $n(\%)$} \\
\hline Female & $287(100)$ & - & $191(72.3)$ & $60(62.5)$ & $34(52.3)$ \\
\hline Male & - & $142(100)$ & $73(27.7)$ & $36(37.5)$ & $31(47.7)$ \\
\hline $\mathrm{BMI}$, mean $(\mathrm{SE}), \mathrm{kg} / \mathrm{m}^{2}$ & $28.2(0.44)$ & $25.4(0.42)$ & $27.6(0.44)$ & $26.8(0.58)$ & $26.7(0.88)$ \\
\hline Duration of infection, mean (SE), years & $10.9(0.32)$ & $12.2(0.49)$ & $11.0(0.34)$ & $10.5(0.58)$ & $13.8(0.66)$ \\
\hline Viral load, mean (SE), $\log _{10}$ copies $/ \mathrm{mL}$ & $4.65(0.05)$ & $4.73(0.07)$ & $4.66(0.06)$ & $4.68(0.09)$ & $4.73(0.10)$ \\
\hline CD $4+$ count, median (range), cells $/ \mathrm{mm}^{3}$ & $210(1,868)$ & $175(2,1125)$ & $179(1,868)$ & $208(1,1125)$ & $249(6,826)$ \\
\hline CDC Class C, $n(\%)$ & $102(35.5)$ & $67(47.2)$ & $111(42.0)$ & $33(34.4)$ & $22(33.8)$ \\
\hline Prior use of $\geq 2$ PIs, $n(\%)$ & $168(58.5)$ & $92(64.8)$ & $156(59.1)$ & $60(62.5)$ & $42(64.6)$ \\
\hline PSS of the OBR ${ }^{\mathrm{b}}$, mean $(\mathrm{SD})$ & $2.0(0.65)$ & $2.0(0.81)$ & $2.0(0.72)$ & $1.9(0.67)$ & $2.0(0.67)$ \\
\hline Hepatitis B surface antigen (positive), $n(\%)$ & $12(4.2)$ & $7(4.9)$ & $15(5.7)$ & $3(3.1)$ & $1(1.5)$ \\
\hline Hepatitis C antibody (positive), $n(\%)$ & $39(13.6)$ & $25(17.6)$ & $41(15.5)$ & $12(12.5)$ & $11(16.9)$ \\
\hline
\end{tabular}

${ }^{\mathrm{a}}$ Two women and two men self-identified as Asian or other and were not included in the analysis by race. ${ }^{\mathrm{b}} \mathrm{By}$ virco TYPE; SE: standard error; BMI: body mass index; CDC: Centers for Disease Control and Prevention; PI: protease inhibitor; PSS: phenotypic susceptibility score; OBR: optimized background regimen; SD: standard deviation.

TABLE 2: Results from the univariate analysis to investigate factors associated with improvements in total FAHI score over 48 weeks.

\begin{tabular}{lc}
\hline Covariate & $P$ value \\
\hline Baseline FAHI & $<.0001$ \\
Baseline CD4+ count & .0052 \\
Confirmed virologic response & .0335 \\
History of psychological illness & .0570 \\
Baseline $\log _{10}$ viral load & .0862 \\
Stage of HIV infection & .1441 \\
Sex & .1979 \\
Presence of etravirine in the OBR & .2062 \\
Alcohol use & .2700 \\
Duration of diagnosis & .2995 \\
Black race versus other & .3490 \\
Incidence of grade 2-4 AE & .4374 \\
Smoker & .5272 \\
Presence of hepatitis C antibody & .5938 \\
Age & .7183 \\
Structured treatment interruption & .7757 \\
Adherence & .8049 \\
Drug use & .9548 \\
\hline
\end{tabular}

FAHI: Functional Assessment of HIV Infection; OBR: optimized background regimen; AE: adverse event.

total FAHI score still improved from baseline to Week 48 (Figure 2(a)) to a similar extent as in the total population.

3.3. Health-Related Quality of Life: Functional Assessment of HIV Infection Subscales. The EWB and PWB subscales showed significant changes from baseline to Week 12, which were maintained through Week 48 for the overall population and across all sex and race subgroups (Table 4). Women
TABLE 3: Factors associated with improvements in total FAHI score over 48 weeks in the final multivariate model.

\begin{tabular}{lc}
\hline Covariate & $P$ value \\
\hline Lower baseline FAHI & $<.0001$ \\
Confirmed virologic response & .0045 \\
Analysis time point ${ }^{\mathrm{a}}$ & .0002 \\
Lower baseline CD4+ count & .0077 \\
\hline${ }^{\mathrm{a}}$ Total FAHI score increases over time; FAHI: Functional Assessment of HIV \\
Infection.
\end{tabular}

and black patients demonstrated the largest changes in EWB compared with the other subgroups. The FGWB and SWB subscales showed small improvements from baseline through Week 48 overall. Changes in the FGWB subscale were only significant for women and black patients (Table 4). The CF subscale values did not change over 48 weeks for the overall population, or for any of the sex and race subgroups.

\section{Discussion}

Many factors can impact the HRQoL of patients with HIV-1, including the tolerability of different ARV agents. Differences in safety/tolerability, pharmacokinetic parameters, and/or efficacy of ARVs could potentially contribute to sex-based or race-based differences in HRQoL scores. To date, studies investigating sex-based and race-based differences in HRQoL for HIV-1-infected patients have reported conflicting results [9-12]. In the GRACE study, HRQoL significantly improved for all participants. Near-maximal improvements were reached by Week 12 and were maintained through Week 48 . When only patients who completed the trial were analyzed, similar improvements in total FAHI score over time were still seen, suggesting that the improvements in the overall 


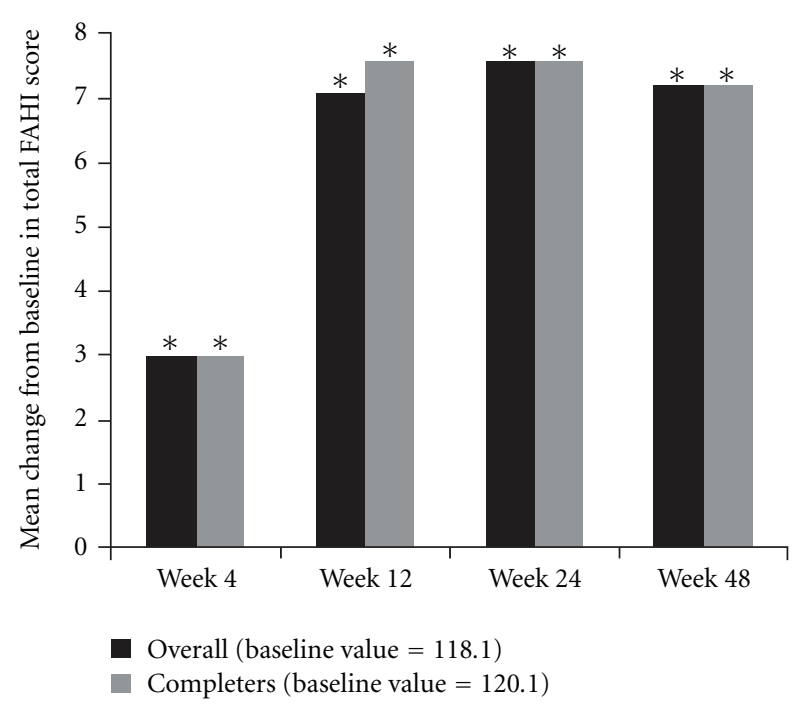

(a)

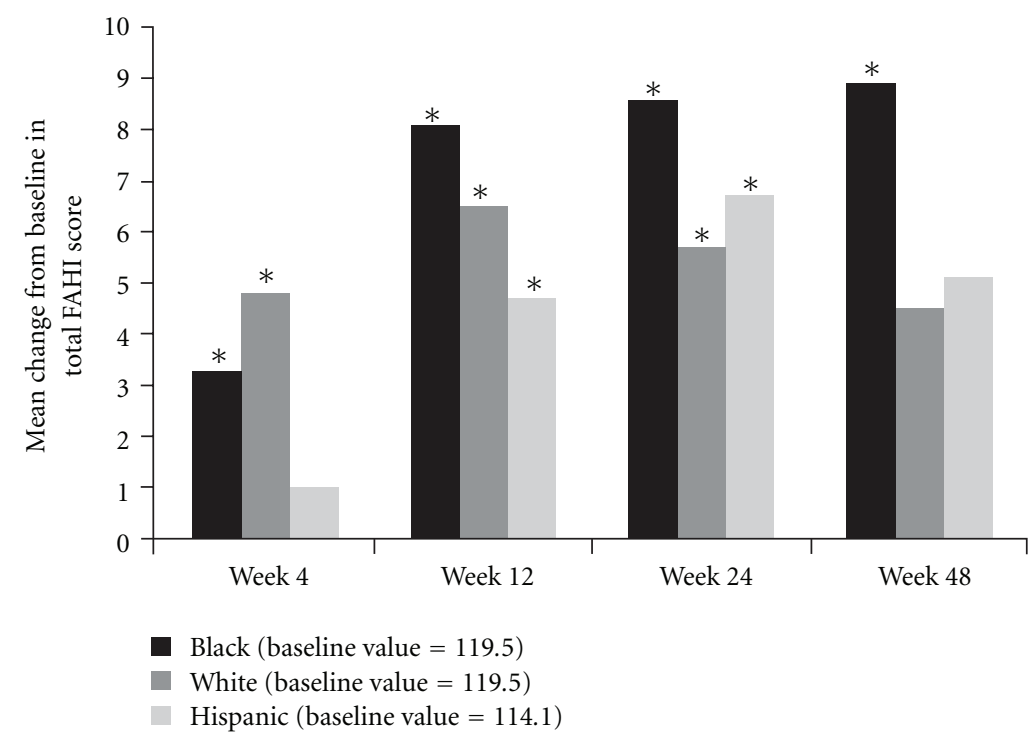

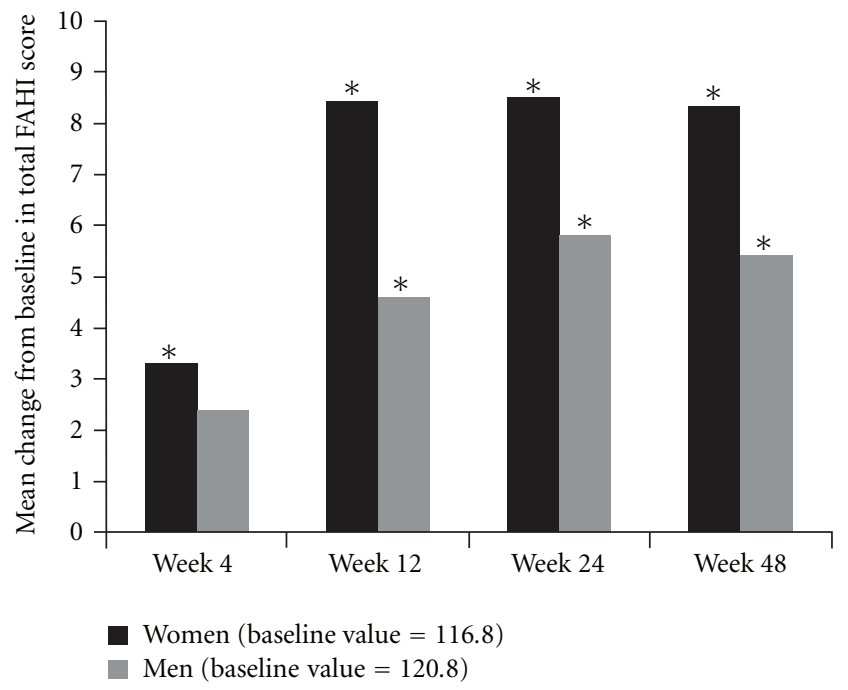

(b)

(c)

FIgURE 2: Mean change from baseline in total FAHI score in (a) the overall population and completers ${ }^{\mathrm{a}}$, (b) by sex, and (c) by race ${ }^{\mathrm{b}}$ (observed). *Statistically significant change from baseline $(P<.05) .{ }^{a}$ Excludes patients who discontinued the trial. ${ }^{b}$ Four patients selfidentified as Asian or other and were not included in the analysis by race due to small sample size; FAHI: functional assessment of HIV infection.

population were not an artifact of patients with low HRQoL scores discontinuing the trial.

All sex and racial/ethnic groups demonstrated improvements in HRQoL over 48 weeks. These results are consistent with those from a previous study of 1178 patients receiving ARV therapy, which also showed that HRQoL improved over time, regardless of sex [11]. In univariate assessments, the largest improvements in total FAHI scores were seen in women and black patients, despite the fact that these two groups both had lower virologic response rates and higher discontinuation rates compared with men and with Hispanic and white patients, respectively. The differences seen here in total FAHI scores were driven by the larger increases in the EWB and FGWB subscales for women and black patients compared with the other subgroups. The post hoc multivariate analysis, however, found that neither black race nor sex was significantly associated with improved total FAHI scores over 48 weeks. Given that sex and race were not associated with improved total FAHI in the multivariate analysis, it is likely that differences in the change in HRQoL by sex and race were at least partially driven by differences in baseline characteristics, such as disease severity. These results are in agreement with those from another study of $287 \mathrm{HIV}$ 1-infected women who completed a HRQoL assessment 
TABLE 4: Mean change from baseline in FAHI questionnaire subscales (observed ${ }^{\mathrm{a}}$ ).

\begin{tabular}{|c|c|c|c|c|c|c|c|c|c|c|c|c|}
\hline & $n$ & Overall & $n$ & Women & $n$ & Men & $n$ & Black & $n$ & Hispanic & $n$ & White \\
\hline \multicolumn{13}{|l|}{ PWB } \\
\hline BL & 424 & 29.4 & 283 & 28.8 & 141 & 30.6 & 262 & 29.5 & 94 & 29.7 & 64 & 29.0 \\
\hline Wk $12 \Delta$ & 359 & $2.6^{\mathrm{b}}$ & 236 & $2.7^{\mathrm{b}}$ & 123 & $2.3^{\mathrm{b}}$ & 218 & $2.6^{\mathrm{b}}$ & 82 & $2.6^{\mathrm{b}}$ & 55 & $2.2^{\mathrm{b}}$ \\
\hline Wk $48 \Delta$ & 298 & $2.2^{\mathrm{b}}$ & 190 & $2.3^{\mathrm{b}}$ & 108 & $2.0^{\mathrm{b}}$ & 176 & $2.3^{\mathrm{b}}$ & 71 & $2.1^{\mathrm{b}}$ & 48 & $1.8^{\mathrm{b}}$ \\
\hline \multicolumn{13}{|l|}{ EWB } \\
\hline $\mathrm{BL}$ & 423 & 25.1 & 283 & 24.5 & 140 & 26.5 & 261 & 25.4 & 94 & 23.3 & 64 & 26.7 \\
\hline Wk $12 \Delta$ & 358 & $3.1^{\mathrm{b}}$ & 235 & $3.6^{\mathrm{b}}$ & 123 & $2.2^{\mathrm{b}}$ & 218 & $3.6^{\mathrm{b}}$ & 82 & $2.1^{\mathrm{b}}$ & 54 & $2.8^{\mathrm{b}}$ \\
\hline Wk $48 \Delta$ & 297 & $3.8^{\mathrm{b}}$ & 190 & $4.5^{\mathrm{b}}$ & 107 & $2.5^{\mathrm{b}}$ & 175 & $4.1^{\mathrm{b}}$ & 71 & $3.8^{\mathrm{b}}$ & 48 & $2.3^{\mathrm{b}}$ \\
\hline \multicolumn{13}{|l|}{ SWB } \\
\hline $\mathrm{BL}$ & 423 & 20.5 & 283 & 20.7 & 140 & 19.9 & 261 & 21.0 & 94 & 18.5 & 64 & 21.3 \\
\hline Wk $12 \Delta$ & 359 & 0.2 & 236 & 0.4 & 123 & -0.2 & 218 & 0.2 & 82 & -0.5 & 55 & 0.9 \\
\hline Wk $48 \Delta$ & 296 & 0.5 & 189 & 0.3 & 107 & 1.0 & 174 & 0.8 & 71 & 0.1 & 48 & 0.6 \\
\hline \multicolumn{13}{|l|}{$\mathrm{CF}$} \\
\hline BL & 423 & 8.2 & 283 & 8.0 & 140 & 8.7 & 261 & 8.3 & 94 & 8.0 & 64 & 8.2 \\
\hline Wk $12 \Delta$ & 359 & 0.1 & 236 & 0.3 & 123 & -0.2 & 218 & 0.2 & 82 & -0.1 & 55 & 0.1 \\
\hline Wk $48 \Delta$ & 296 & -0.1 & 189 & 0.1 & 107 & -0.4 & 174 & 0.0 & 71 & -0.3 & 48 & -0.2 \\
\hline \multicolumn{13}{|l|}{ FGWB } \\
\hline $\mathrm{BL}$ & 423 & 34.9 & 283 & 34.8 & 140 & 35.2 & 261 & 35.4 & 94 & 34.5 & 64 & 34.3 \\
\hline Wk $12 \Delta$ & 359 & $1.1^{\mathrm{b}}$ & 236 & $1.4^{\mathrm{b}}$ & 123 & 0.6 & 218 & $1.5^{\mathrm{b}}$ & 82 & 0.6 & 55 & 0.5 \\
\hline Wk $48 \Delta$ & 297 & 0.7 & 190 & 1.0 & 107 & 0.3 & 175 & $1.5^{\mathrm{b}}$ & 71 & -0.6 & 48 & 0.1 \\
\hline
\end{tabular}

${ }^{\mathrm{a}}$ The $n$ value varies for each parameter. ${ }^{\mathrm{b}}$ Significant change $(P<.05)$; FAHI: Functional Assessment of HIV Infection; PWB: physical well-being; BL: baseline; Wk: week; $\Delta$ : change from baseline; EWB: emotional well-being; SWB: social well-being; CF: cognitive functioning; FGWB: functional and global well-being.

during their current treatment regimen. This study also found that race had no bearing on HRQoL [10]; however, changes in HRQoL over time were not assessed.

Lower baseline FAHI scores, lower baseline CD4+ cell counts, and confirmed virologic response were identified as significantly associated with improved total FAHI scores over 48 weeks. CD4+ cell count and general disease state have previously been associated with absolute HRQoL scores in other studies $[10,12,13]$. Higher CD4+ cell counts have been linked to higher HRQoL scores in women and men $[10,13]$, whereas the presence of AIDS has been linked to lower self-perceived physical health scores in both sexes [12]. The DUET study, which investigated the efficacy and safety of etravirine in treatment-experienced patients, also demonstrated an association between baseline FAHI score and CD4+ cell counts and improvement in FAHI scores over 24 weeks [14].

Results from this study suggest that DRV/r-based, optimized, ARV therapy over 48 weeks results in significant improvement in self-perceived HRQoL, regardless of sex and race. However, due to the single-arm design of this study, we cannot conclude whether this improvement is better or worse than those associated with other ARV regimens. Previous studies of sex-based and race-based differences in HRQoL scores have demonstrated conflicting results, with some studies noting differences in overall HRQoL between the sexes or races and other studies reporting no differences [9-12]. These studies, however, are limited due to low proportions of women or people of color and/or the enrollment of women only, with no male group for comparison. In contrast, the large proportion of women enrolled in GRACE, along with a sufficient number of men and the large proportion of people of color, may allow more clinically meaningful conclusions to be drawn regarding sexbased and race-based differences in HRQoL.

Although the overall conclusions from previous studies about sex-based or race-based differences in HRQoL are contradictory, previous studies do suggest that factors affecting HRQoL scores may vary between sexes. One study showed that longer duration of infection and low levels of social support were linked to lower HRQoL scores in women, whereas difficulty taking ARV tablets, intravenous drug use, and low levels of social support were associated with lower HRQoL scores in men [12]. These sex-associated differences may make it possible to tailor HIV treatments and treatment support to women and men, thus maximizing their overall QoL.

In this trial, confirmed virologic response was found to be significantly associated with improved total FAHI scores. In the GRACE trial, women and black patients had lower response rates and higher discontinuation rates than the other sex and race subgroups [5]. Based on this, lower improvements in FAHI scores in these two groups may have been expected. However, the opposite was observed, with women and black patients demonstrating the largest improvements in FAHI scores over 48 weeks. These contrary results suggest the need to measure alternative outcomes in clinical trials, as virologic response alone may not be 
wholly indicative of a patient's overall improvement while on ARV therapy. The GRACE study was designed with patient support in mind, with opportunities to apply for sponsorship for site-specific recruitment and retention activities [15]. These activities may have contributed to the subjects' comfort level with both treatment and study participation and may have led to a greater sense of attachment to site staff and care delivery. It is likely that factors distinct from virologic response may be influencing patients' overall HRQoL, particularly for women and black patients, and these should be investigated in the future.

Interestingly, we found that patients with lower baseline FAHI scores were significantly more likely to discontinue than patients with higher baseline HRQoL scores. Although data are lacking with regard to the association between HRQoL and retention of patients in clinical trials, other studies have linked lower HRQoL scores to decreased survival of HIV-1-infected subjects [2-4]. Furthermore, measures of HRQoL in a large veteran outpatient population have also been shown to predict survival more generally [16]. In the future, it may be possible to identify patients with a higher risk of discontinuation based on their baseline HRQoL scores; these patients could then be more closely monitored, potentially improving retention in care. This approach deserves further study.

\section{Conclusions}

The sex-based and race-based differences in the improvements in total FAHI score and FAHI subscales may provide insight into the subtly different effects of HIV-1 infection and treatment on HRQoL in different populations. The data from GRACE on HRQoL by sex and race may provide valuable information into methods resulting in future optimization of treatments and retention in care for specific populations of HIV-infected patients.

\section{Acknowledgments}

J. Currier has received research grants from Tibotec, Merck, Schering-Plough, and Theratechnologies, acted as an advisor for Tibotec, Bristol-Myers Squibb, Merck, and GlaxoSmithKline, and received honoraria from GlaxoSmithKline. K. Squires has received grant/research support from Biocryst, Gilead Sciences, GlaxoSmithKline, Merck, Tibotec, and ViiV, acted as a consultant for GlaxoSmithKline and Merck, been an advisory board member for Bristol-Myers Squibb, Gilead Sciences, GlaxoSmithKline, Merck, Tibotec, and Tobira, and is a DSMB member with Pfizer. J. Feinberg has received grant support from Tibotec, Roche, BristolMyers Squibb, GlaxoSmithKline, and Pfizer and served as a speaker and/or consultant for Tibotec, Bristol-Myers Squibb, Merck, GlaxoSmithKline, and Pfizer. M. Saag has received research grant support from Tibotec, Bristol-Myers Squibb, Merck, Boehringer Ingelheim, ViiV, and Gilead and acted as a consultant/investigator for Tibotec, Bristol-Myers Squibb, Merck, Boehringer Ingelheim, ViiV, and Gilead. B. Coate and R. Ryan are employees of Tibotec Inc. J. Mrus is an employee of Tibotec Therapeutics. The authors would like to thank the patients and their families, the staff and principal investigators, and the study sites for their participation in the trial. The authors would like to acknowledge Gilead for supplying emtricitabine, tenofovir disoproxil fumarate, and emtricitabine/tenofovir disoproxil fumarate. The authors would additionally like to acknowledge internal study support staff, as well as Cali Howitt, Ph.D., Medicus International New York, for her editorial assistance.

\section{References}

[1] F. J. Palella Jr., K. M. Delaney, A. C. Moorman et al., "Declining morbidity and mortality among patients with advanced human immunodeficiency virus infection. HIV outpatient study investigators," The New England Journal of Medicine, vol. 338, no. 13, pp. 853-860, 1998.

[2] W. E. Cunningham, S. Crystal, S. Bozzette, and R. D. Hays, "The association of health-related quality of life with survival among persons with HIV infection in the United States," Journal of General Internal Medicine, vol. 20, no. 1, pp. 21-27, 2005.

[3] I. M. de Boer-van der Kolk, M. A. G. Sprangers, J. M. Prins, C. Smit, F. de Wolf, and P. T. Nieuwkerk, "Health-related quality of life and survival among HIV-infected patients receiving highly active antiretroviral therapy: a study of patients in the AIDS therapy evaluation in the Netherlands (ATHENA) cohort," Clinical Infectious Diseases, vol. 50, no. 2, pp. 255-263, 2010.

[4] W. C. Mathews and S. May, "EuroQol (EQ-5D) measure of quality of life predicts mortality, emergency department utilization, and hospital discharge rates in HIV-infected adults under care," Health and Quality of Life Outcomes, vol. 5, article 5, 2007.

[5] J. Currier, D. A. Bridge, D. Hagins et al., "Sex-based outcomes of Darunavir-Ritonavir therapy: a single-group trial," Annals of Internal Medicine, vol. 153, no. 6, pp. 349-357, 2010.

[6] A. H. Peterman, D. Cella, F. Mo, and N. McCain, "Psychometric validation of the revised functional assessment of human immunodeficiency virus infection (FAHI) quality of life instrument," Quality of Life Research, vol. 6, no. 6, pp. 572584, 1997.

[7] M. Viala-Danten, D. Dubois, H. Gilet, S. Martin, K. Peeters, and D. Cella, "Psychometric evaluation of the functional assessment of HIV Infection (FAHI) questionnaire and its usefulness in clinical trials," Quality of Life Research, vol. 19, no. 8, pp. 1215-1227, 2010.

[8] K. Smith, F. Garcia, R. Ryan, R. Falcon, A. Tennenberg, and J. Mrus, "GRACE (Gender, Race And Clinical Experience): outcomes by race at week 48," in Proceedings of the 49th Interscience Conference on Antimicrobial Agents and Chemotherapy, San Francisco, Calif, USA, Poster H-918, 12-15 September 2009.

[9] L. S. B. Figuero, P. B. Luque, T. P. Martin, M. G. Sagrado, and J. M. E. Bouza, "Assessment of factors influencing health-related quality of life in HIV-infected patients," HIV Medicine, vol. 12, no. 1, pp. 22-30, 2011.

[10] K. A. McDonnell, A. C. Gielen, A. W. Wu, P. O’Campo, and R. Faden, "Measuring health related quality of life among women living with HIV," Quality of Life Research, vol. 9, no. 8, pp. 931940, 2000.

[11] J. M. Mrus, P. L. Williams, J. Tsevat, S. E. Cohn, and A. W. Wu, "Gender differences in health-related quality of life in patients 
with HIV/AIDS," Quality of Life Research, vol. 14, no. 2, pp. 479-491, 2005.

[12] I. R. Pérez, A. Olry de Labry Lima, L. S. del Castillo, J. R. Baño, M. A. L. Ruz, and A. del Arco Jimenez, "No differences in quality of life between men and women undergoing HIV antiretroviral treatment. Impact of demographic, clinical and psychosocial factors," AIDS Care, vol. 21, no. 8, pp. 943-952, 2009.

[13] E. G. Bing, R. D. Hays, L. P. Jacobson et al., "Health-related quality of life among people with HIV disease: results from the multicenter AIDS cohort study," Quality of Life Research, vol. 9, no. 1, pp. 55-63, 2000.

[14] D. Cella, H. Gilet, M. Viala-Danten, K. Peeters, D. Dubois, and S. Martin, "Effects of etravirine versus placebo on health-related quality of life in treatment-experienced HIV patients as measured by the functional assessment of human immunodeficiency virus infection (FAHI) questionnaire in the DUET trials," HIV Clinical Trials, vol. 11, no. 1, pp. 1827, 2010.

[15] R. Falcon, D. A. Bridge, J. Currier et al., "Recruitment and retention of diverse populations in antiretroviral clinical trials: practical applications from the GRACE (Gender, Race And Clinical Experience) study," Journal of Womens Health, vol. 20, no. 7, pp. 1043-1050, 2011.

[16] K. B. DeSalvo, V. S. Fan, M. B. McDonell, and S. D. Fihn, "Predicting mortality and healthcare utilization with a single question," Health Services Research, vol. 40, no. 4, pp. 12341246, 2005. 


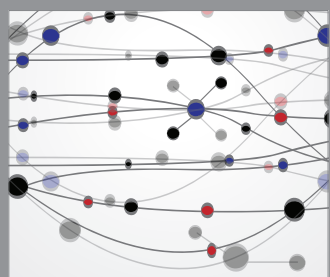

The Scientific World Journal
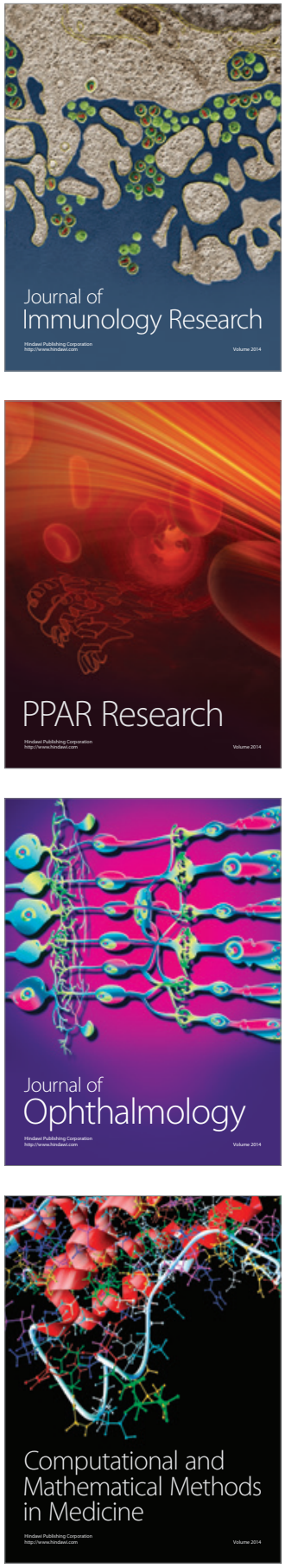

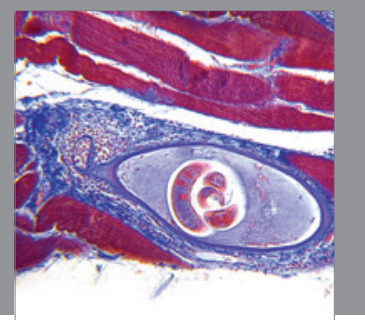

Gastroenterology

Research and Practice
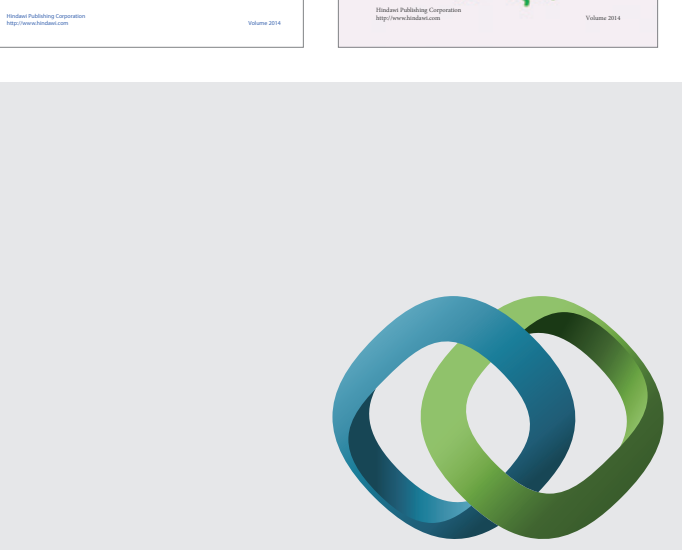

\section{Hindawi}

Submit your manuscripts at

http://www.hindawi.com
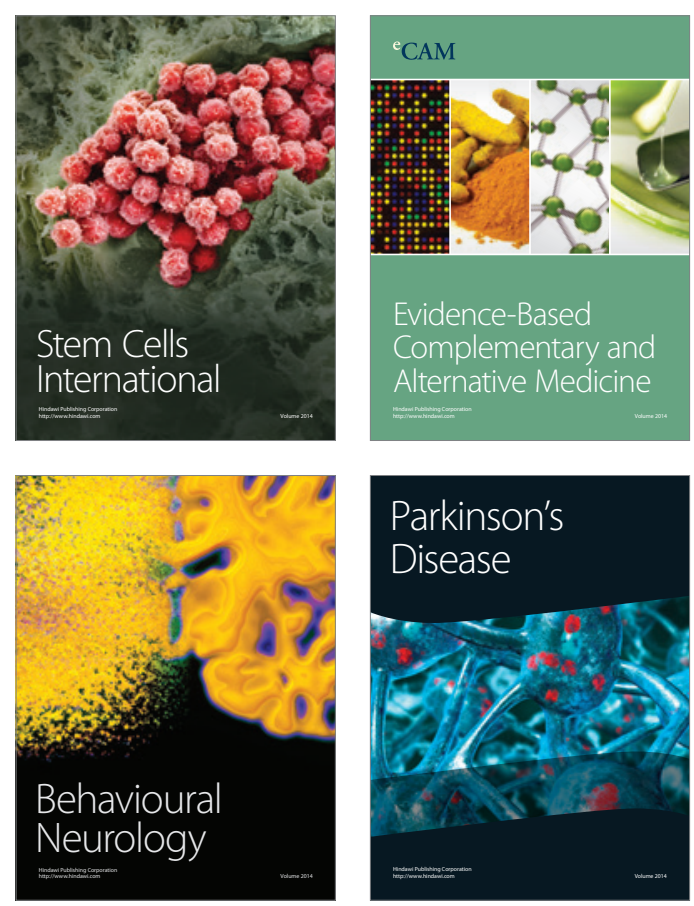

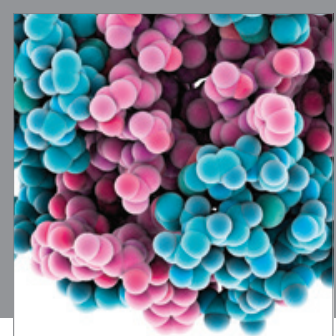

Journal of
Diabetes Research

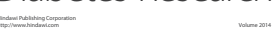

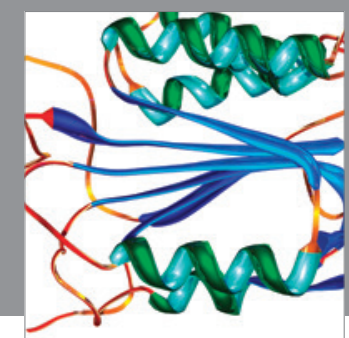

Disease Markers
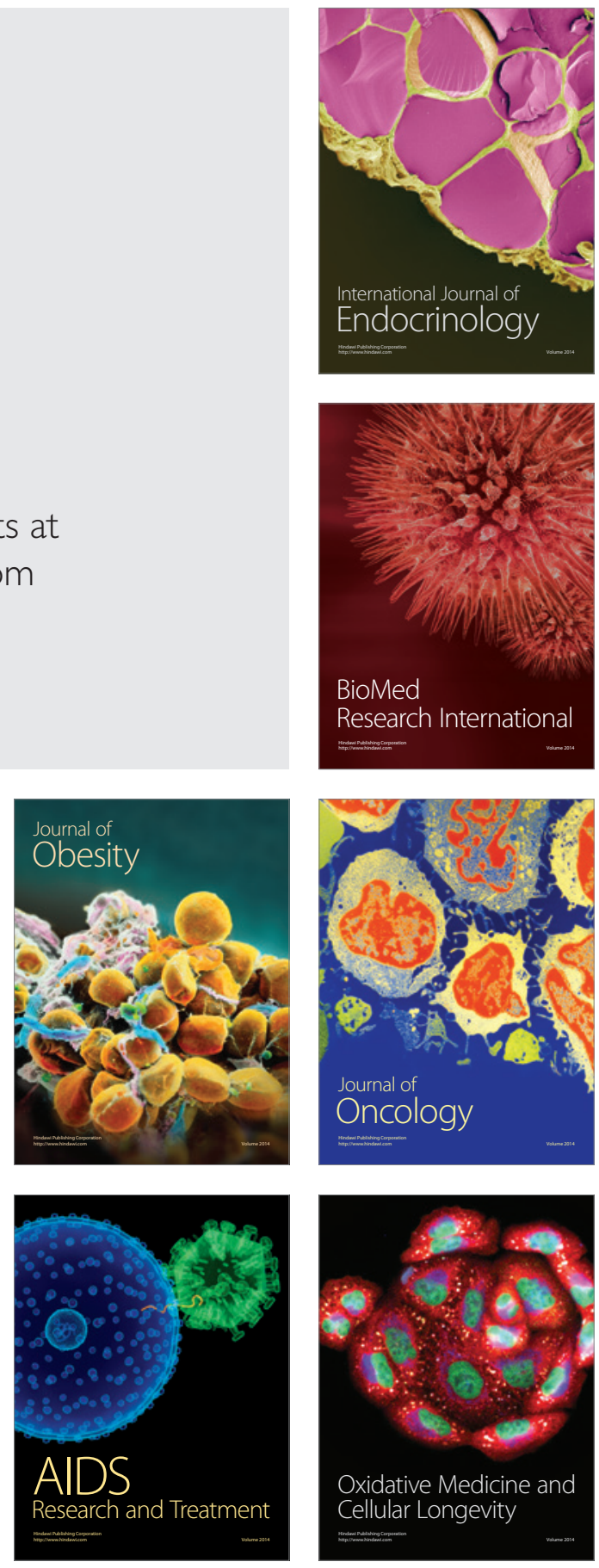